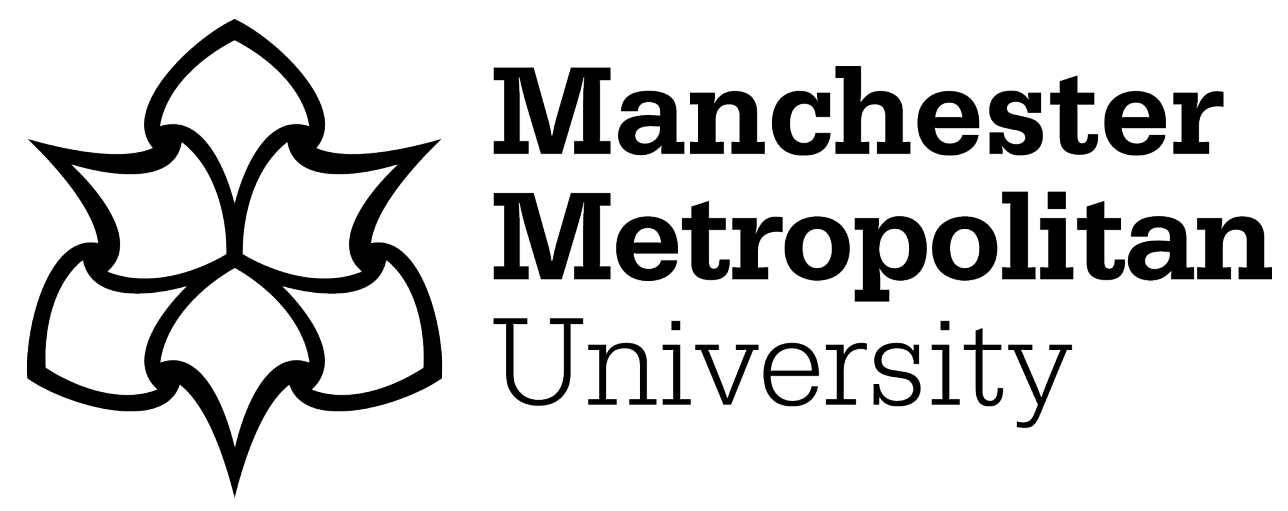

Goulden, N, Khusnulina, A, Davis, NJ, Bracewell, RM, Bokde, AL, McNulty, JP and Mullins, PG (2014) The salience network is responsible for switching between the default mode network and the central executive network: Replication from DCM. Neurolmage, 99. pp. 180-190. ISSN 1053-8119

Downloaded from: https://e-space.mmu.ac.uk/617647/

Version: Accepted Version

Publisher: Elsevier

DOI: https://doi.org/10.1016/j.neuroimage.2014.05.052

Please cite the published version 


\title{
The salience network is responsible for switching between the default mode network and the central executive network: Replication from DCM
}

\author{
Nia Goulden ${ }^{\mathrm{a}, *}$, Aygul Khusnulina ${ }^{\mathrm{a}}$, Nicholas J. Davis ${ }^{\mathrm{b}}$, Robert M. Bracewell ${ }^{\mathrm{a}}$, Arun L. Bokde ${ }^{\mathrm{c}}$, \\ Jonathan P. McNulty ${ }^{\mathrm{d}}$, Paul G. Mullins ${ }^{\mathrm{a}}$ \\ a Bangor University, Bangor, UK \\ b Swansea University, Swansea, UK \\ c Trinity College Dublin, Dublin, Ireland \\ ${ }^{\mathrm{d}}$ University College Dublin, Dublin, Ireland
}

\section{A R T I C L E I N F O}

Article history:

Accepted 18 May 2014

Available online 24 May 2014

\begin{abstract}
A B S T R A C T
With the advent of new analysis methods in neuroimaging that involve independent component analysis (ICA) and dynamic causal modelling (DCM), investigations have focused on measuring both the activity and connectivity of specific brain networks. In this study we combined DCM with spatial ICA to investigate network switching in the brain. Using time courses determined by ICA in our dynamic causal models, we focused on the dynamics of switching between the default mode network (DMN), the network which is active when the brain is not engaging in a specific task, and the central executive network (CEN), which is active when the brain is engaging in a task requiring attention. Previous work using Granger causality methods has shown that regions of the brain which respond to the degree of subjective salience of a stimulus, the salience network, are responsible for switching between the DMN and the CEN (Sridharan et al., 2008). In this work we apply DCM to ICA time courses representing these networks in resting state data. In order to test the repeatability of our work we applied this to two independent datasets. This work confirms that the salience network drives the switching between default mode and central executive networks and that our novel technique is repeatable.
\end{abstract}

Crown Copyright (c) 2014 Published by Elsevier Inc. All rights reserved.

\section{Introduction}

The development of many new analysis methods for functional neuroimaging data such as functional connectivity, independent component analysis (ICA) and effective connectivity methods including dynamic causal modelling (DCM) has lead to a lot of work focused on brain network activity and connectivity. An important ability of the human brain is to be able to rapidly switch between different tasks; it is therefore of interest to study interactions between different networks of the brain to better understand the mechanism behind switching between different tasks.

Dynamic causal modelling (DCM) as a method was introduced for effective connectivity analyses in 2003 (Friston et al., 2003). Typically several hypothesised models are specified and Bayesian model selection (BMS) is used to infer the model which represents the best fit to the data

\footnotetext{
* Corresponding author at: Brigantia Building, Penrallt Road, Bangor, Gwynedd, LL57 2AS, UK.

E-mail addresses: niagoulden@yahoo.co.uk (N. Goulden), pspaa6@bangor.ac.uk (A. Khusnulina), n.j.davis@swansea.ac.uk (N.J. Davis), m.bracewell@bangor.ac.uk (R.M. Bracewell), arun.bokde@tcd.ie (A.L. Bokde), jonathan.mcnulty@ucd.ie (J.P. McNulty), p.mullins@bangor.ac.uk (P.G. Mullins).
}

(Penny et al., 2004). Recent advances in methodology mean it is now possible to compare families of DCMs (Penny et al., 2010), to use a random effects method for model selection (Stephan et al., 2009) and to specify nonlinear and stochastic models (Li et al., 2011; Stephan et al., 2008). The introduction of nonlinear modelling means it is now possible to test for modulations of regions on connections i.e. how regions influence connection strengths between other brain regions, providing a more realistic model of brain physiology. Stochastic modelling means that the input into the model is not deterministic, more accurately accounting for noise (Daunizeau et al., 2012) and allowing the application of DCM to resting state data, which was not previously thought possible. Stochastic DCMs also include random fluctuations in the state equations that better accounts for spontaneous neuronal fluctuations. Conventional DCM, in contrast, uses deterministic differential equations, and as a result does not account for spontaneous neuronal fluctuations or state noise.

Another method that has been used extensively to examine brain networks is independent component analysis (ICA) (Bell and Sejnowski, 1995; Comon, 1994; Hyvarinen and Oja, 2000). Whilst it is possible to apply both spatial and temporal ICA, we are using spatial ICA for our analysis. ICA of fMRI data separates the data into spatially independent patterns of activity and therefore can identify brain networks engaged in a task without the use of a predefined model. ICA 


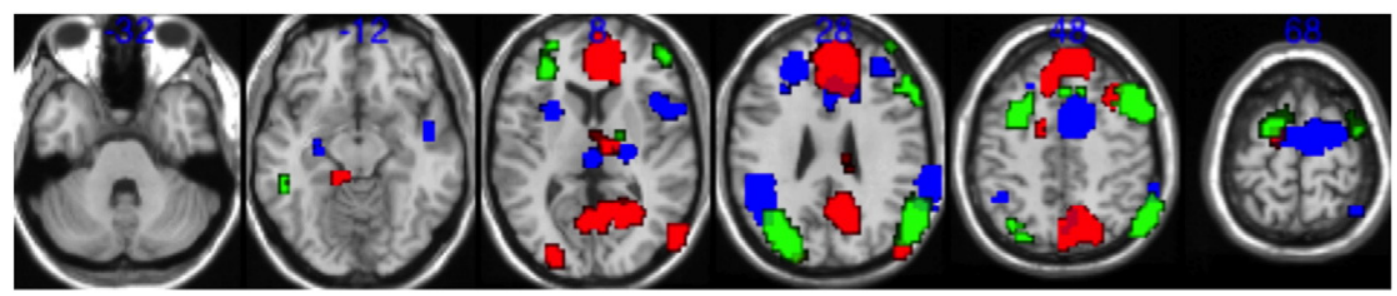

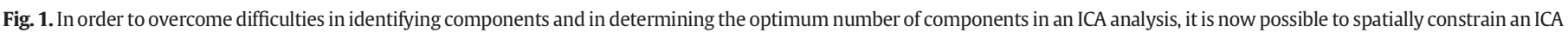

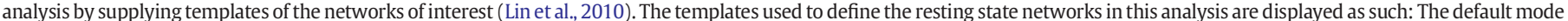
network (DMN) is shown in red, the salience network (SN) in blue and the central executive network (CEN) in green. We display slices at $\mathrm{z}=-22,-12,8,28,48$ and 68 .

has also been applied to resting state data (Lee et al., 2012) and has shown differences in functional network connectivity with age (Stevens et al., 2009) and in patients with schizophrenia (Yu et al., 2011). Resting state networks found in this fashion have been shown to be reproducible, with many networks having a high interclass correlation coefficient in a repetition experiment (Chou et al., 2012; Damoiseaux et al., 2006; Shehzad et al., 2009). In order to overcome difficulties in identifying components of interest and in determining the optimum number of components in an ICA analysis, it is now possible to spatially constrain an ICA analysis to provide the components of interest (Lin et al., 2010) by supplying templates of the networks of interest. This is particularly useful if the research question relates to specific networks of the brain.

Sridharan et al. (2008) recently used Granger causality to examine the relationship between different networks. One of the networks they studied is the default mode network (DMN). This network is consistently observed in ICA analyses of resting state data and task deactivation studies (Beckmann et al., 2005; Raichle et al., 2001). The DMN comprises the posterior cingulate (BA 23 and 31), posterior parietal cortex (BA 7, 39 and 40) and the ventromedial prefrontal cortex (Buckner et al., 2008). The central executive network (CEN) comprises the dorsolateral prefrontal cortex and posterior parietal cortex and is engaged when a cognitively demanding task requiring attention is being performed (Fox et al., 2006). The third network is the salience network (SN) which includes the ventrolateral prefrontal cortex (VLPFC) and anterior insula (jointly referred to as the fronto-insular cortex; FIC) and the anterior cingulate cortex (ACC) (Seeley et al., 2007). The salience network responds to the degree of subjective salience, whether cognitive, homeostatic, or emotional. The networks are displayed in Fig. 1, where the DMN is shown in red, the SN in blue and the CEN in green. Based on this work and others, a model has been proposed of the function of the insula, including bottom-up detection of salient events, switching between other networks to facilitate access to attention and working memory upon detection of a salient event, interaction of the anterior and posterior insula for autonomic reactivity to salient stimuli and strong functional coupling with the anterior cingulate to facilitate rapid access to the motor system (Menon and Uddin, 2010).

Recently relationships between brain networks have been increasingly studied with the advent of methods such as functional network connectivity (Jafri et al., 2008), which examines temporal relations between components. We wanted to explore whether it would be possible to use DCM to examine the relationship between different ICA components from resting state data, and therefore examine the relationship between different networks of the brain, specifically network switching.

The aim of this work is to further examine the network switching demonstrated by Sridharan et al. (2008), and to do so by applying a novel technique using nonlinear DCM with stochastic modelling of ICA components from resting state data. In order to demonstrate repeatability of our findings we also apply this analysis to an independent dataset.

\section{Methods}

We used two different datasets for this analysis. The first dataset was acquired at Bangor University, and the second was from an open access database.

\section{Participants - data acquired in Bangor University}

Participants were 20 healthy male participants, mean age 29.6 (SD 11.2) and 22 healthy female participants, mean age 35.8 (SD 12.7). The local research ethics committee approved the study.

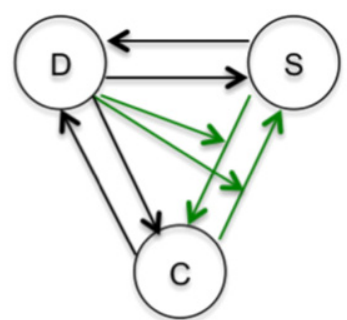

DMN

Modulations

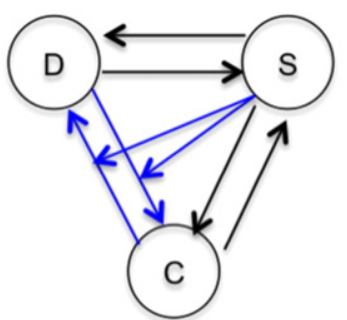

SN Modulations

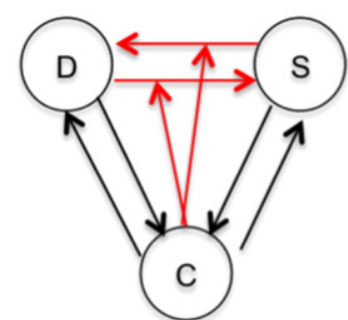

CEN

Modulations

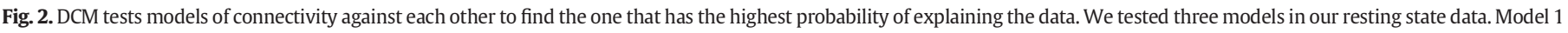

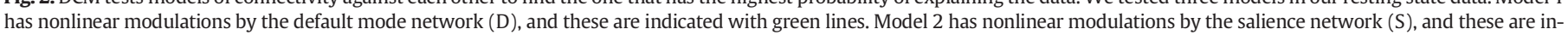
dicated in blue lines. Model 3 has nonlinear modulations by the central executive network $(\mathrm{C})$, and these are indicated with red lines. 

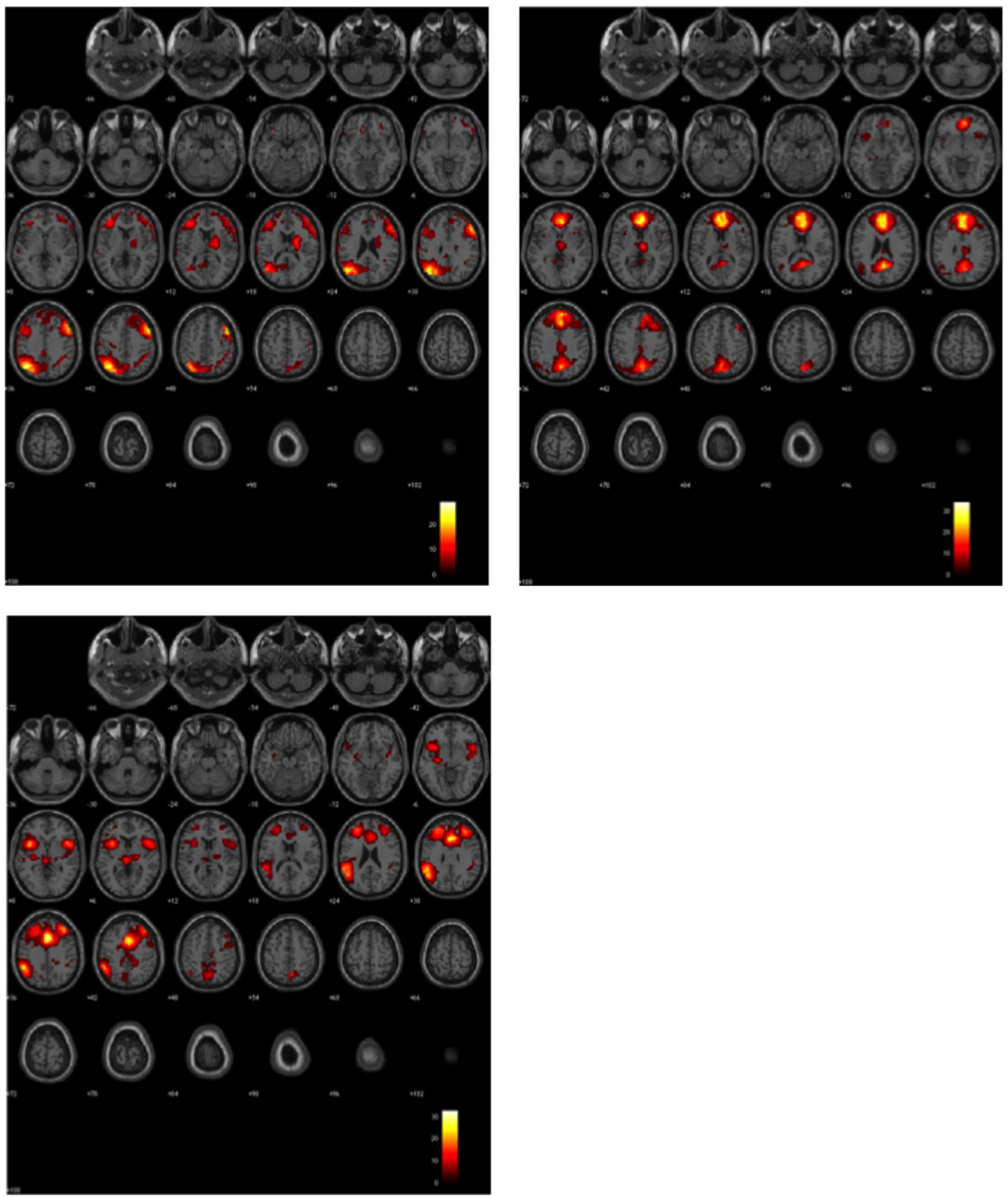

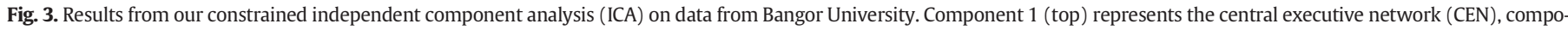

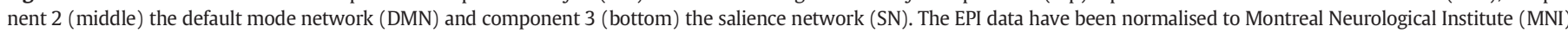
space, the bottom slice is $Z=-72$, each subsequent slice increases $Z$ by $6 \mathrm{~mm}$ up to $Z=108$. The colour bars represent the $t$-value of the BOLD signal.

\section{Participants - NITRC 1000 Functional Connectomes Project}

These are freely available resting state data on the NITRC Web site (http://fcon_1000.projects.nitrc.org/indi/pro/nki.html). On this Web site new data are uploaded as they are acquired on a five weekly basis. We included the data acquired during weeks 1 through 35 and the participants aged between 20 and 30 in order to be comparable to previous work and to find the typical result in a population of young adults. The dataset comprised 24 male participants, mean age 23.2 (SD 2.7) and 20 female participants, mean age 24.6 (SD 3.44). 

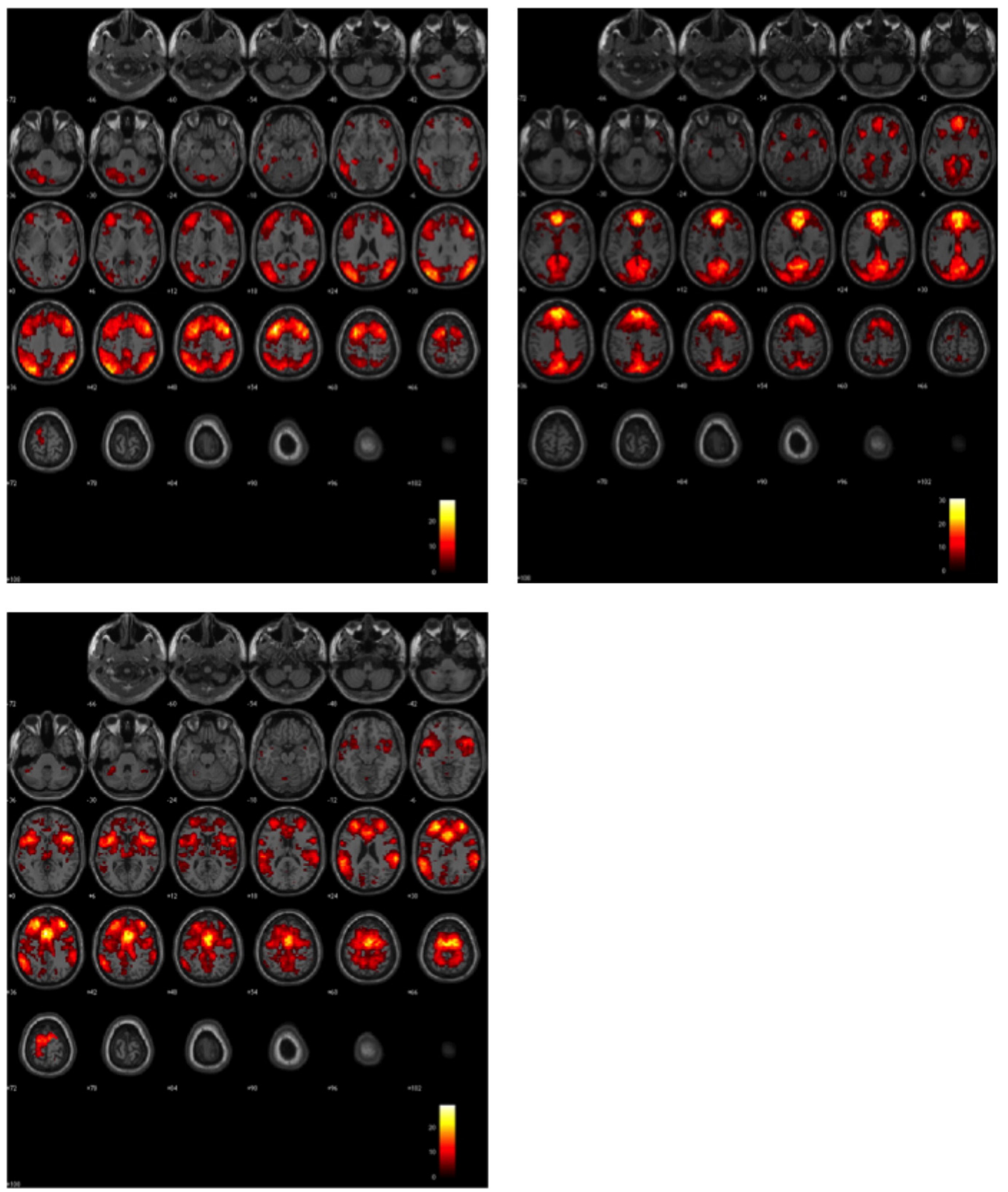

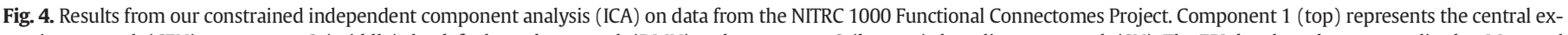

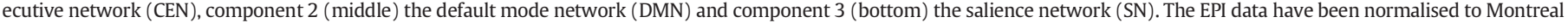
Neurological Institute (MNI) space, the bottom slice is $\mathrm{Z}=-72$, each subsequent slice increases $\mathrm{Z}$ by $6 \mathrm{~mm}$ up to $\mathrm{Z}=108$. The colour bars represent the t-value of the bold signal.

\section{Resting state sessions}

For the resting state scans participants rested in the scanner with their eyes open, they were not given any specific instructions other than to keep as still as possible. Nothing was displayed to them during this time and other than scanner noise there were no additional auditory stimuli. For the Bangor dataset resting state data were collected for five minutes, whilst with the NITRC data, resting state scans were acquired for ten minutes. 

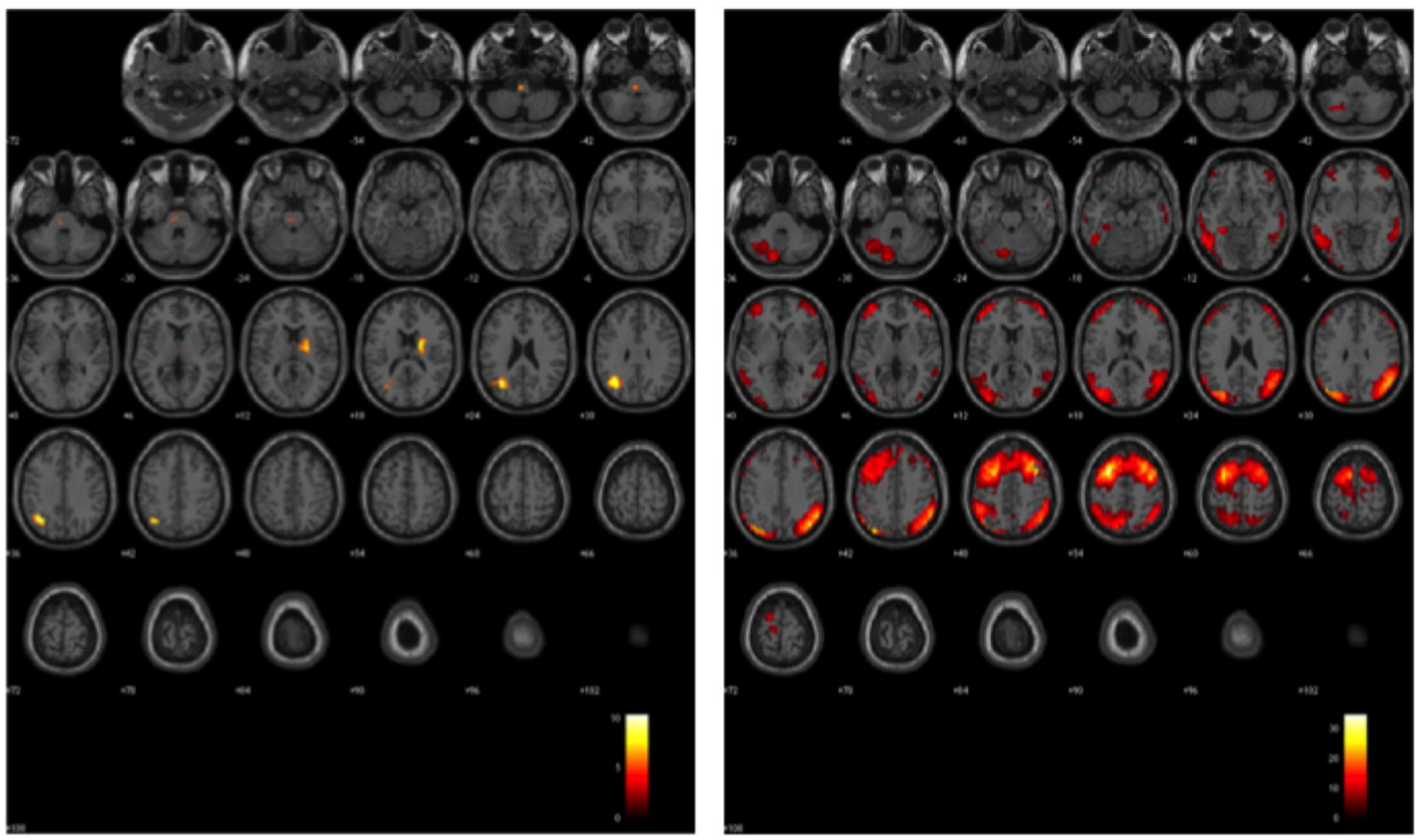

Fig. 5. Comparison of the CEN component between the two datasets. The left image displays Bangor $>$ NITRC and the right image displays Bangor $<$ NITRC. All images are thresholded at $\mathrm{p}<0.05$ (FWE) with an extent threshold of 50, so all clusters are significant at $\mathrm{p}<0.0001$ (FWE). The EPI data have been normalised to Montreal Neurological Institute (MNI) space, the bottom slice is $Z=-72$, each subsequent slice increases $Z$ by $6 \mathrm{~mm}$ up to $Z=108$. The colour bars represent the t-value of the BOLD signal.
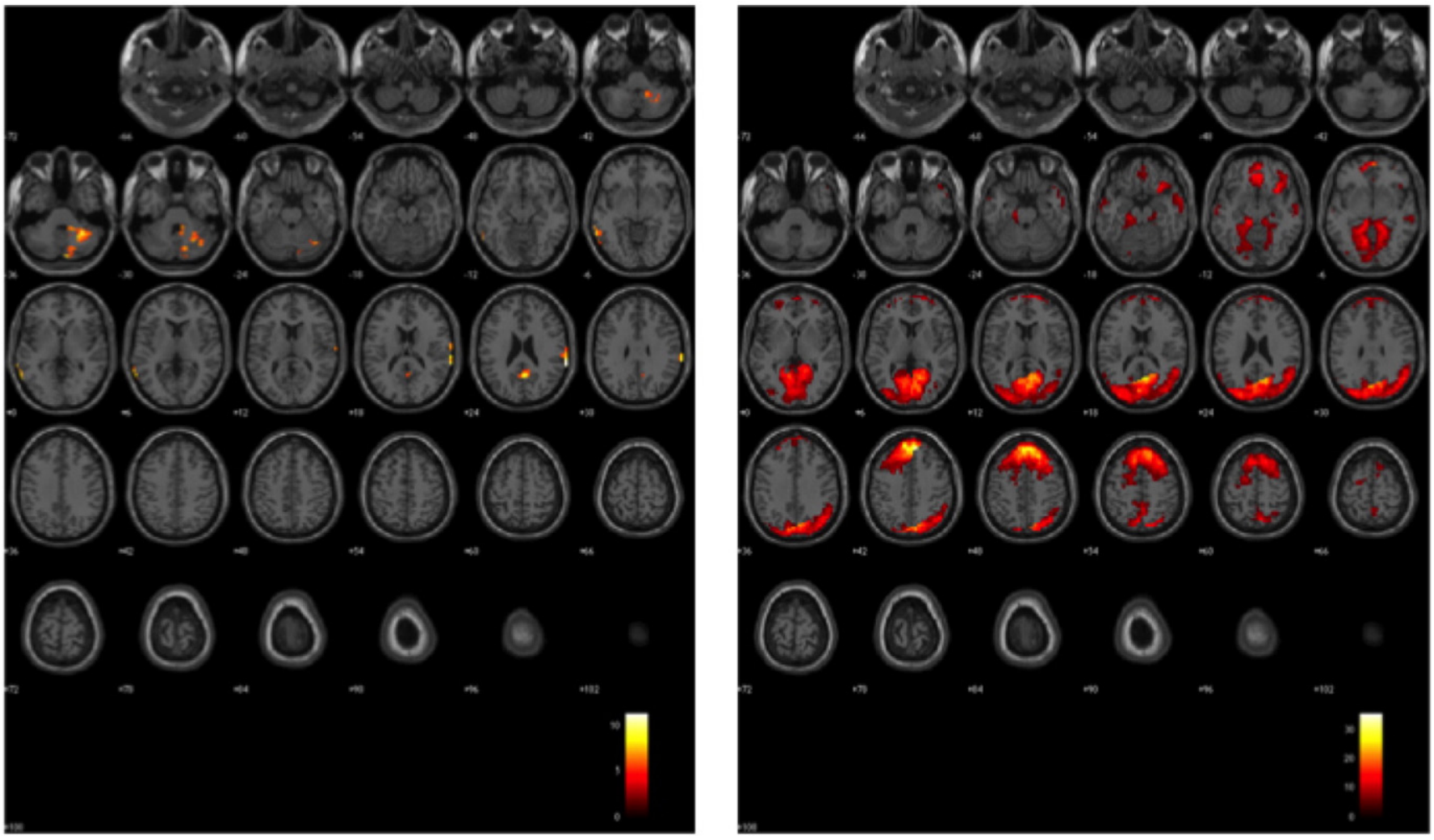

Fig. 6. Comparison of the DMN component between the two datasets. The left image displays Bangor $<$ NITRC and the right image displays Bangor $<$ NITRC. All images are thresholded at $\mathrm{p}<0.05$ (FWE) with an extent threshold of 50, so all clusters are significant at $\mathrm{p}<0.0001$ (FWE). The EPI data have been normalised to Montreal Neurological Institute (MNI) space, the bottom slice is $Z=-72$, each subsequent slice increases $Z$ by $6 \mathrm{~mm}$ up to $Z=108$. The colour bars represent the $t$-value of the bold signal. 

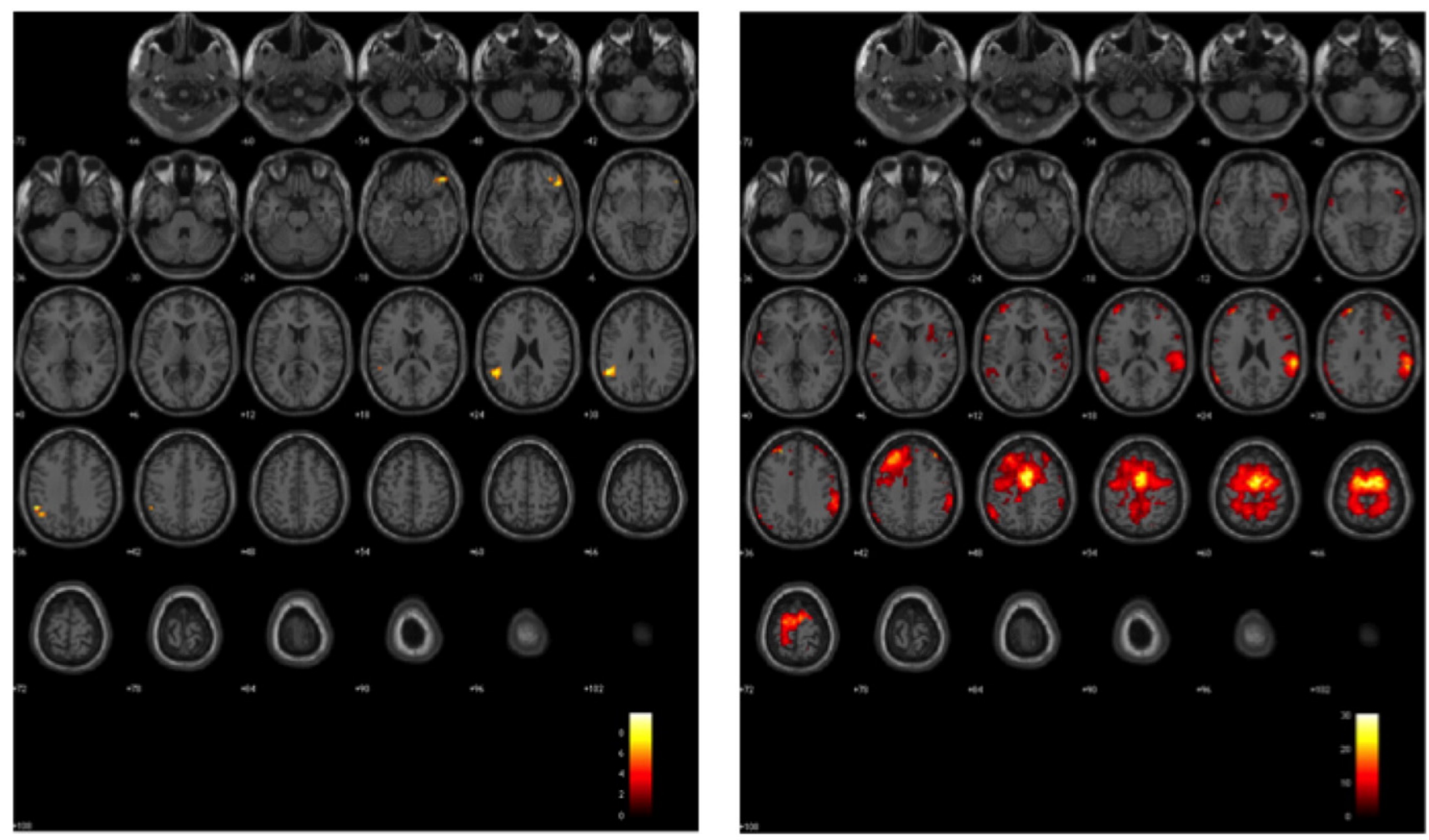

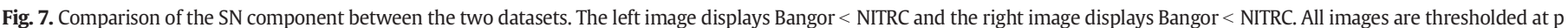

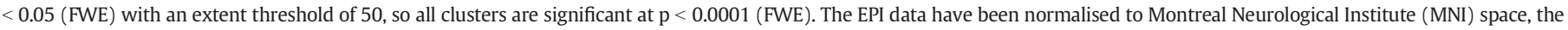
bottom slice is $Z=-72$, each subsequent slice increases $Z$ by $6 \mathrm{~mm}$ up to $Z=108$. The colour bars represent the $t$-value of the bold signal.

\section{Magnetic resonance imaging - data acquired in Bangor University}

Images were acquired using a single-shot echo-planar pulse sequence on a 3 T Phillips Achieva scanner (Eindhoven, Netherlands). Each three-dimensional volume comprised 35 contiguous axial slices in an ascending order (TR/TE $=2000 / 30 \mathrm{~ms}, 3.0 \mathrm{~mm}$ by $3.0 \mathrm{~mm}$ inplane resolution, slice thickness $3.0 \mathrm{~mm}$ ). A high-resolution T1weighted structural image was also collected for each participant to assist with registration to standard stereotactic space (TR/TE $=14 / 4 \mathrm{~ms}$, $1.0 \mathrm{~mm}$ by $1.0 \mathrm{~mm}$ in-plane resolution, slice thickness $1.0 \mathrm{~mm}$ ).

During the resting state condition, 150 volumes were acquired. No volumes were discarded before analysis, as the Philips scanner automatically excluded T1 magnetisation equilibrium volumes.

Magnetic resonance imaging - NITRC 1000 Functional Connectomes Project

Images were acquired using a single-shot echo-planar pulse sequence on a $3 \mathrm{~T}$ Siemens Magnetom Trio Tim scanner. Each threedimensional volume comprised 38 axial slices in an interleaved order $(\mathrm{TR} / \mathrm{TE}=2500 / 30 \mathrm{~ms}, 3.0 \mathrm{~mm}$ by $3.0 \mathrm{~mm}$ in-plane resolution, slice thickness $3.0 \mathrm{~mm}$ ). A high-resolution T1-weighted structural image was also collected for each participants to assist with registration to standard stereotactic space (TR/TE $=8.2 / 3.5 \mathrm{~ms}, 1.0 \mathrm{~mm}$ by $1.0 \mathrm{~mm}$ in-plane resolution, slice thickness $1.0 \mathrm{~mm}$ ).

During the resting state condition, 260 volumes were acquired. In order to exclude T1 magnetisation equilibrium effects the first 10 time points were excluded. For consistency between datasets we retained the following 150 time points for analysis.

Image analysis

The preprocessing was carried out using Statistical Parametric Mapping (SPM8) (http://www.fil.ion.ucl.ac.uk/spm). Data were slice timing corrected and realigned to the first volume. The anatomical image was coregistered to the mean functional image and then segmented using the 'New Segment' method into grey matter, white matter and cerebrospinal fluid (CSF). Functional data were then normalised to standard space and smoothed using DARTEL (Ashburner, 2007). A groupspecific structural template was created for both datasets to perform this analysis. For functional connectivity analyses of resting state data it is important to regress out motion and physiological parameters, however this is not necessary for ICA since it mixes the spatial information and makes it more difficult for the ICA to separate artifact from signal. The nature of the ICA analysis means that any noise will be placed into separate components and it is therefore not necessary to perform this step beforehand. We therefore use the data preprocessed in the usual way for fMRI.

\section{Independent component analysis}

Independent component analysis was carried out using the Group ICA of fMRI Toolbox (GIFT, http://mialab.mrn.org/software/gift/). Constrained ICA was applied for this analysis in order to identify the networks of interest. This means that templates are supplied and the constrained ICA method identifies independent components within the data that provide a close match to the supplied templates. Constrained ICA is a semiblind ICA method, which means that prior information is incorporated into the estimation process (Lin et al., 2010). This also resolves some ambiguity associated with blind ICA and overcomes the problem of deciding how many components to define. The semiblind ICA algorithm assumes $M$ source signals, and has spatial information for $L$ sources of interest. Only the $L$ sources from the mixtures will be extracted in a predefined order. Reference signals $r_{1}, \ldots, r_{L}$ are constructed from the spatial information about the $L$ sources of interest, and a closeness measure between extracted signal and 
Table 1

Results of comparing the CEN component extracted for the Bangor dataset and the NITRC dataset. We present the clusters that were found in this table. Results are thresholded at $\mathrm{p}<0.05$ (FWE) with an extent threshold of 50, so all clusters are significant at $\mathrm{p}<0.0001$ (FWE). This table lists the results for the comparisons Bangor $>$ NITRC and also Bangor $<$ NITRC.

\begin{tabular}{|c|c|c|}
\hline Cluster Maxima & Label & Number of voxels in cluster \\
\hline \multicolumn{3}{|l|}{ Bangor $>$ NITRC } \\
\hline \multirow[t]{3}{*}{$-39-6036$} & Left angular gyrus & \multirow[t]{3}{*}{219} \\
\hline & Left middle occipital gyrus & \\
\hline & Left inferior parietal lobe & \\
\hline \multirow[t]{3}{*}{$21-318$} & Right caudate & \multirow[t]{3}{*}{106} \\
\hline & Right thalamus & \\
\hline & Right putamen & \\
\hline \multicolumn{3}{|l|}{ Bangor $<$ NITRC } \\
\hline \multirow[t]{13}{*}{-271554} & Left middle frontal gyrus & \multirow[t]{13}{*}{4366} \\
\hline & Right middle frontal gyrus & \\
\hline & Left superior frontal gyrus & \\
\hline & Right superior frontal gyrus & \\
\hline & Left precentral gyrus & \\
\hline & Left supplementary motor area & \\
\hline & Right supplementary motor area & \\
\hline & Left medial frontal gyrus & \\
\hline & Right medial frontal gyrus & \\
\hline & Left inferior frontal gyrus & \\
\hline & Left paracentral lobule & \\
\hline & Right precentral gyrus & \\
\hline & Right inferior frontal gyrus & \\
\hline \multirow[t]{20}{*}{$-33-7839$} & Left middle occipital gyrus & \multirow[t]{20}{*}{4647} \\
\hline & Right angular gyrus & \\
\hline & Right middle temporal gyrus & \\
\hline & Left middle temporal gyrus & \\
\hline & Right middle occipital gyrus & \\
\hline & Left inferior parietal lobe & \\
\hline & Left cerebellum crus 1 & \\
\hline & Left superior parietal lobe & \\
\hline & Right inferior parietal lobe & \\
\hline & Right supramarginal gyrus & \\
\hline & Left inferior temporal gyrus & \\
\hline & Right superior parietal & \\
\hline & Left superior occipital gyrus & \\
\hline & Left precuneus & \\
\hline & Left cerebellum crus 2 & \\
\hline & Right superior occipital gyrus & \\
\hline & Left inferior occipital gyrus & \\
\hline & Right inferior temporal gyrus & \\
\hline & Right superior temporal gyrus & \\
\hline & Right precuneus & \\
\hline \multirow[t]{2}{*}{$-30-39-12$} & Left fusiform gyrus & \multirow[t]{2}{*}{63} \\
\hline & Left parahippocampal gyrus & \\
\hline
\end{tabular}

reference signal is defined to constrain the learning of the mixing matrix.

The GIFT toolbox supplies Resting State Network Templates, which are templates of components found from resting state data. Templates for the DMN, SN and CEN were constructed from these templates for use in the constrained ICA. Constrained ICA was then applied to our data using these templates. The time courses from the ICA analysis were used for performing the DCM analysis. Using ICA to extract representative networks has been done previously and been found to provide better representations of networks than taking the average or first eigenvariate of a large template (Craddock et al., 2012; Shirer et al., 2012; Smith et al., 2011; Yu et al., 2012).

We chose to use constrained ICA with these templates in order to obtain the best possible independent component to represent our networks, avoid issues with deciding on the number of components and bias in the analysis from having to decide on which component to use.

In our case we have three sources of interest, one for each component/network we are interested in, therefore $\mathrm{L}$ is three. For our analysis the closeness measure was set to 0.08 , which is the default and minimum value for this parameter.
Table 2

Results of comparing the DMN component extracted for the Bangor dataset and the NITRC dataset. We present the clusters that were found in this table. Results are thresholded at $\mathrm{p}<0.05$ (FWE) with an extent threshold of 50, so all clusters are significant at $\mathrm{p}<0.0001$ (FWE). This table lists the results for the comparisons Bangor $>$ NITRC and also Bangor $<$ NITRC.

\begin{tabular}{|c|c|c|}
\hline Cluster maxima & Label & Number of voxels in cluster \\
\hline \multicolumn{3}{|l|}{ Bangor $>$ NITRC } \\
\hline \multirow[t]{3}{*}{$69-3924$} & Right supramarginal gyrus & 60 \\
\hline & Right postcentral gyrus & \\
\hline & Right superior temporal gyrus & \\
\hline \multirow[t]{2}{*}{$-63-573$} & Left middle temporal gyrus & 67 \\
\hline & Left inferior temporal gyrus & \\
\hline \multirow[t]{3}{*}{$33-51-36$} & Right cerebellum crus 1 & 212 \\
\hline & Right cerebellum & \\
\hline & Right fusiform gyrus & \\
\hline \multirow[t]{4}{*}{$6-5724$} & Right precuneus & 50 \\
\hline & Left precuneus & \\
\hline & Right posterior cingulate & \\
\hline & Left posterior cingulate & \\
\hline \multirow[t]{3}{*}{$9-87-36$} & Right cerebellum crus 2 & 55 \\
\hline & Right cerebellum & \\
\hline & Right cerebellum crus 1 & \\
\hline \multicolumn{3}{|l|}{ Bangor $<$ NITRC } \\
\hline \multirow[t]{10}{*}{$-6-5139$} & Left superior frontal gyrus & 2899 \\
\hline & Right superior frontal gyrus & \\
\hline & Left medial frontal gyrus & \\
\hline & Left middle frontal gyrus & \\
\hline & Right medial frontal gyrus & \\
\hline & Right middle frontal gyrus & \\
\hline & Right supplementary motor area & \\
\hline & Left supplementary motor area & \\
\hline & Right inferior frontal gyrus & \\
\hline & Right insula & \\
\hline \multirow[t]{18}{*}{$9-6315$} & Right calcarine fissure & 5625 \\
\hline & Left middle occipital gyrus & \\
\hline & Left calcarine fissure & \\
\hline & Left lingual gyrus & \\
\hline & Right lingual gyrus & \\
\hline & Left cuneus & \\
\hline & Right angular gyrus & \\
\hline & Right cuneus & \\
\hline & Right middle occipital gyrus & \\
\hline & Right superior occipital gyrus & \\
\hline & Left superior occipital gyrus & \\
\hline & Right precuneus & \\
\hline & Right middle temporal gyrus & \\
\hline & Left precuneus & \\
\hline & Right superior parietal lobe & \\
\hline & Left fusiform gyrus & \\
\hline & Left parahippocampal gyrus & \\
\hline & Right inferior parietal lobe & \\
\hline \multirow[t]{4}{*}{$603-18$} & Right middle temporal gyrus & 175 \\
\hline & Right middle temporal pole & \\
\hline & Right superior temporal gyrus & \\
\hline & Right superior temporal pole & \\
\hline \multirow[t]{2}{*}{$-60-6-15$} & Left middle temporal gyrus & 65 \\
\hline & Left superior temporal gyrus & \\
\hline
\end{tabular}

\section{Dynamic causal modelling}

DCM12 analysis was performed using SPM12b (http://www.fil.ion. ucl.ac.uk/spm). Three models were specified for each participant, all with fully connected intrinsic models and no input entering the system. The first model specified nonlinear modulation of the DMN on both the bidirectional connections between the SN and the CEN. The second model specified nonlinear modulation of the SN on both the bidirectional connections between the DMN and the CEN. The third model specified nonlinear modulation of the CEN on both the bidirectional connections between the DMN and the SN. The models are illustrated in Fig. 2. These models test whether it is the DMN driving switching between the SN and CEN, the SN driving the switching between DMN and CEN or the CEN driving switching between DMN and SN. Stochastic 
DCM was used to estimate the models, which employs non-linear stochastic differential equations.

\section{Results}

\section{Independent component analysis}

The results of the constrained ICA analysis for the Bangor University data are displayed in Fig. 3. Component 1 (top left) is the CEN, component 2 (top right) the DMN and component 3 (bottom left) the SN. In order to test how well our extracted components represent the networks we correlated our binary masks used in the constrained ICA analysis with the extracted component to test how many voxels correspond in the mask and result. Performing a correlation with the CEN mask used in the analysis there is a $32.6 \%$ correlation with the derived CEN component. There is a $54.6 \%$ correlation between the DMN mask and the derived DMN component. The SN derived component has a $52.9 \%$ correlation with the SN mask.

The results of the constrained ICA analysis for the NITRC 1000 Functional Connectomes Project data are displayed in Fig. 4. Component 1 (top left) is the CEN, component 2 (top right) the DMN and component 3 (bottom left) the SN. The constrained ICA analysis provided a $45.0 \%$ correlation between the derived CEN component and the CEN mask. There is a $58.7 \%$ correlation between the derived DMN component and the DMN mask. The derived SN component has a $49.8 \%$ correlation with the SN mask.

Differences in the components between the two datasets are displayed in Figs. 5-7, all p $<0.05$ (FWE), extent threshold of 50. The results are also documented in Tables 1-3. In this test we are comparing the components we found in both groups using an independent samples t-test for differences. In our case the null hypothesis is no difference between the components for both datasets.

When comparing the CEN component there are two clusters where the Bangor dataset displays greater activity than the NITRC dataset. There is one cluster in the parietal lobe and the angular gyrus, the other is in the caudate, thalamus and putamen. The NITRC dataset displays greater activity than the Bangor dataset in three clusters when comparing the CEN component. The results include some of the frontal, temporal parietal and occipital lobes.

Five clusters display greater activity in the Bangor dataset than the NITRC dataset when comparing the DMN component. The first clusters cover mostly the temporal lobe with some cerebellum. In four clusters there is greater activity in the NITRC dataset than the Bangor dataset when comparing the DMN component. The results cover parts of the frontal parietal and occipital lobes.

The Bangor dataset displays greater activity than the NITRC dataset in two clusters when comparing the SN component. These results cover parts of the frontal and temporal lobes. There are four clusters where the NITRC dataset displays greater activity than the Bangor dataset when comparing the SN component. The clusters cover mostly the frontal lobe with some parietal, temporal and occipital lobe differences.

For further details of these results see Tables 1,2 and 3.

\section{Dynamic causal modelling}

The results of the BMS analysis for the Bangor University data are displayed in Fig. 8. This figure displays the results of the BMS at the individual model level. The figures show the expected posterior model probability and the exceedance probability, which is the probability that a model is more likely than any other model in model space. Recently the protected exceedance probability has been introduced as a summary statistic (Rigoux et al., 2014). This quantifies the probability that any one model is more frequent than the others, above and beyond chance. The winning model from the BMS analysis is model 2, the model with nonlinear modulations by the salience network. The exceedance
Table 3

Results of comparing the SN component extracted for the Bangor dataset and the NITRC dataset. We present the clusters that were found in this table. Results are thresholded at $\mathrm{p}<0.05$ (FWE) with an extent threshold of 50 , so all clusters are significant at $\mathrm{p}<0.0001$ (FWE). This table lists the results for the comparisons Bangor $>$ NITRC and also Bangor $<$ NITRC.

\begin{tabular}{|c|c|c|}
\hline Cluster maxima & Label & Number of voxels in cluster \\
\hline \multicolumn{3}{|l|}{ Bangor $>$ NITRC } \\
\hline \multirow[t]{5}{*}{$-51-4833$} & Left supramarginal gyrus & 134 \\
\hline & Left angular gyrus & \\
\hline & Left inferior parietal lobe & \\
\hline & Left middle temporal gyrus & \\
\hline & Left superior temporal gyrus & \\
\hline \multirow[t]{2}{*}{$4839-18$} & Right inferior frontal gyrus & 62 \\
\hline & Right middle frontal gyrus & \\
\hline \multicolumn{3}{|l|}{ Bangor $<$ NITRC } \\
\hline \multirow[t]{20}{*}{$6-363$} & Right supplementary motor area & 6170 \\
\hline & Left middle frontal gyrus & \\
\hline & Left supplementary motor area & \\
\hline & Left superior frontal gyrus & \\
\hline & Right superior frontal gyrus & \\
\hline & Right supramarginal gyrus & \\
\hline & Left precentral gyrus & \\
\hline & Right middle frontal gyrus & \\
\hline & Right postcentral gyrus & \\
\hline & Left precuneus & \\
\hline & Left postcentral gyrus & \\
\hline & Right precentral gyrus & \\
\hline & Right superior temporal gyrus & \\
\hline & Left paracentral lobe & \\
\hline & Right precuneus & \\
\hline & Left medial frontal gyrus & \\
\hline & Left middle cingulate & \\
\hline & Right paracentral lobe & \\
\hline & Right superior parietal lobe & \\
\hline & Left superior parietal lobe & \\
\hline \multirow{6}{*}{$-57-5424$} & Left middle temporal gyrus & 345 \\
\hline & Left inferior parietal lobe & \\
\hline & Left superior temporal gyrus & \\
\hline & Left angular gyrus & \\
\hline & Left supramarginal gyrus & \\
\hline & Left middle occipital gyrus & \\
\hline \multirow[t]{5}{*}{-5766} & Left inferior frontal gyrus & 121 \\
\hline & Left superior temporal pole & \\
\hline & Left superior temporal gyrus & \\
\hline & Left precentral gyrus & \\
\hline & Left postcentral gyrus & \\
\hline \multirow[t]{3}{*}{33186} & Right insula & 78 \\
\hline & Right putamen & \\
\hline & Right inferior frontal gyrus & \\
\hline
\end{tabular}

probabilities for the three models are $0.2162,0.5138$ and 0.2700 respectively. The protected exceedance probabilities are $0.3121,0.3749$ and 0.3130 respectively.

The results of the BMS analysis for the NITRC 1000 Functional Connectomes Project data are displayed in Fig. 9. The winning model from the BMS analysis is the model with nonlinear modulations by the salience network. The exceedance probabilities for each model are $0.0282,0.9385$ and 0.0333 respectively. The protected exceedance probabilities are $0.0684,0.9252$ and 0.0064 respectively.

The results of Bayesian parameter averaging for both datasets are displayed in Table 4 . The intrinsic connections all have a posterior probability larger than 0.95 for both datasets. The posterior probabilities for the nonlinear modulations are not as high, however they are larger than 0.7 . The results of an independent samples t-test are also presented here. Using a threshold of $\mathrm{p}<0.001$ there are significant differences in the intrinsic connectivity between the DMN and the CEN, however there are no significant differences in the nonlinear modulations.

\section{Discussion}

This work has confirmed the finding of Sridharan et al. (2008) that the SN is key for switching between the CEN and the DMN. We have 

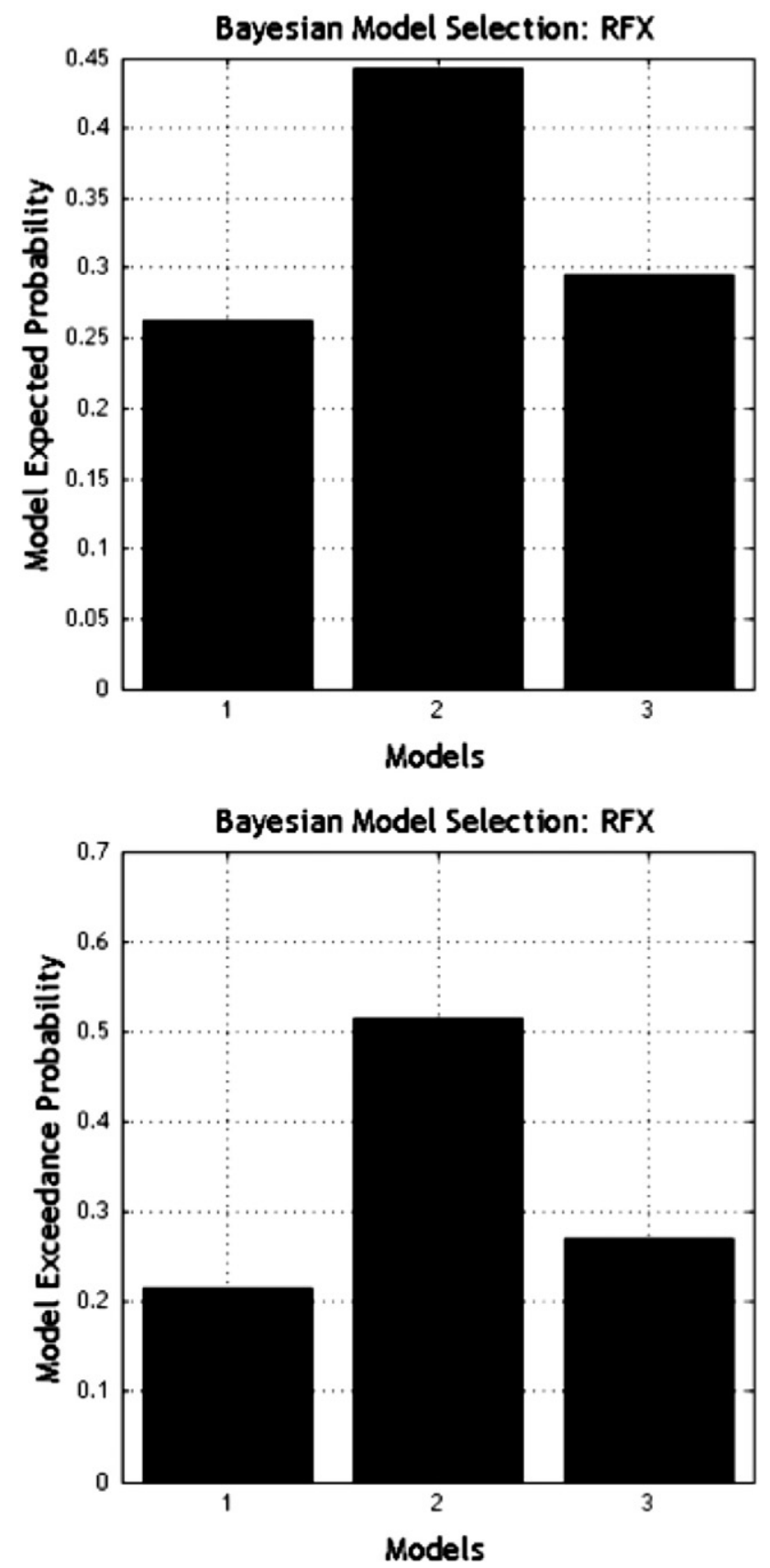

Fig. 8. Results of Bayesian Model Selection for the data from Bangor University showing that model 2, the model with salience network modulating connections between the default mode network and the central executive network, fitted the data best compared to models 1 and 3. (Model 1: the default mode network modulates the connections between the Salience Network and the Central Executive Network. Model 3 the central executive network modulates the connections between the default mode network and salience network.) These results suggest that DCM can be used on resting state data to investigate network modulations.

obtained this result using resting state data; Sridharan and colleagues saw this effect with resting state data and also with task data. The novelty of our work is in demonstrating the same effect using a different modelling technique, DCM as opposed to Granger causality, further validating the role of the SN in switching between CEN and DMN. Our novel approach, to extract the ICA time courses to represent the entire network, rather than look at regions of interest from the networks, also allowed for independent verification, and shows that the result is not dependent on initial seed-point or ROI selection.
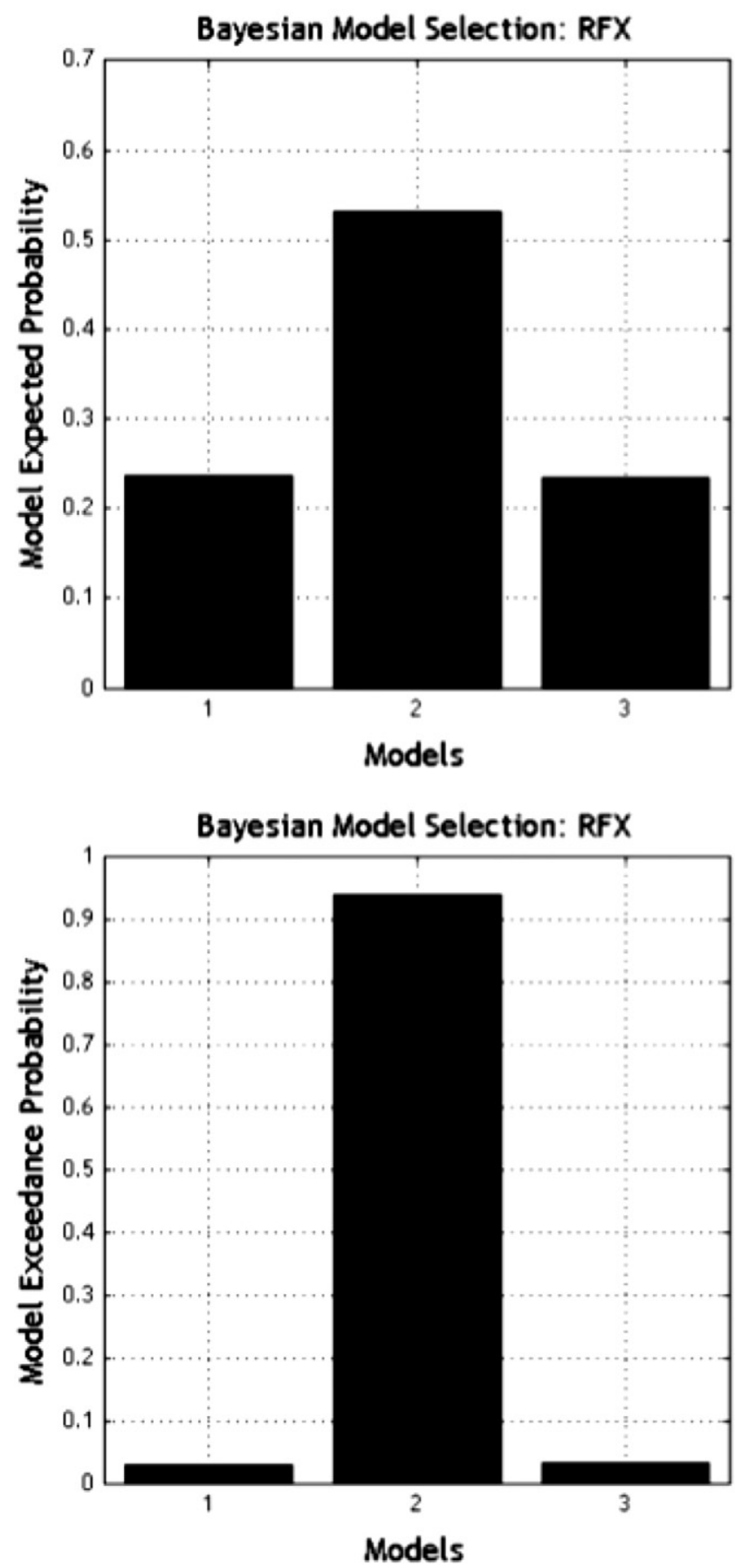

Fig. 9. Results of Bayesian Model Selection for the data from the NITRC 1000 Functional Connectomes Project. Model 2, the model with salience network modulating connections between the default mode network and the central executive network, was again the best fit to the data, outperforming both of the other model. (Model 1: the default mode network modulates the connections between the salience network and the central executive network. Model 3 where the central executive network modulates the connections between the default mode network and salience network.) Combined with the results in Fig. 5, these results confirm that DCM can be used on resting state data to investigate network modulations.

We chose to use a constrained ICA analysis in order to obtain the best representation of each network we were interested in studying. This overcomes issues with deciding on the number of components for the ICA decomposition and potential bias in having to choose components. Using this method if two groups are to be compared then the ICA can be performed separately for each group without bias and this will capture variability in the networks between different groups. We chose to use 


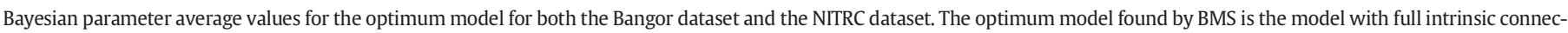

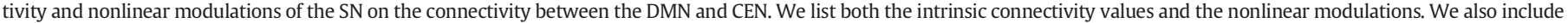
the results of an independent samples t-test for differences between the datasets and the p-value for the t-test.

\begin{tabular}{|c|c|c|c|}
\hline & Bangor dataset & NITRC dataset & \\
\hline Parameter - intrinsic connections & Connection strength (posterior probability) & Connection strength (posterior probability) & t-test ( $\mathrm{p}$-value) \\
\hline $\mathrm{DMN} \rightarrow \mathrm{SN}$ & $0.0287(1.0000)$ & $-0.0064(1.0000)$ & $-3.3889(0.0037)$ \\
\hline $\mathrm{DMN} \rightarrow \mathrm{CEN}$ & $0.0418(1.0000)$ & $0.0409(1.0000)$ & $-5.1049(<0.0001)$ \\
\hline $\mathrm{SN} \rightarrow \mathrm{DMN}$ & $0.0277(1.0000)$ & $0.0012(0.9667)$ & $-3.1431(0.0018)$ \\
\hline $\mathrm{SN} \rightarrow \mathrm{CEN}$ & $-0.0004(0.9978)$ & $-0.0159(1.0000)$ & $3.0698(0.0818)$ \\
\hline $\mathrm{CEN} \rightarrow \mathrm{DMN}$ & $0.0423(1.0000)$ & $0.0461(1.0000)$ & $-5.4845(<0.0001)$ \\
\hline $\mathrm{CEN} \rightarrow \mathrm{SN}$ & $-0.0163(1.0000)$ & $-0.0097(1.0000)$ & $1.8232(0.0045)$ \\
\hline Parameter - nonlinear modulations & Connection strength (posterior probability) & Connection strength (posterior probability) & \\
\hline $\mathrm{SN} \rightarrow(\mathrm{DMN} \rightarrow \mathrm{CEN})$ & $-0.0448(0.7517)$ & $0.0779(0.8036)$ & $-0.9509(0.8504)$ \\
\hline $\mathrm{SN} \rightarrow(\mathrm{CEN} \rightarrow \mathrm{DMN})$ & $0.0877(0.9124)$ & $0.0561(0.7287)$ & $0.1912(0.3444)$ \\
\hline
\end{tabular}

nonlinear DCM in order to study the network switching. We chose this approach because it is possible to directly test for the effect of a network on the connectivity on other networks, demonstrating that the network is responsible for changing the connectivity and therefore network switching. We also made use of stochastic DCM in order to allow us to analyse resting state data. Nonlinear DCM provides the advantage that it is possible to study non-stationarities in resting state connectivity without assumptions regarding window length, as in a sliding window correlation analysis.

Anatomical connectivity within and outside the salience network has been studied previously (Craig, 2002; Critchley et al., 2004; Mesulam, 1998; Mesulam and Mufson, 1982; Mufson and Mesulam, 1982). The ACC and the insula, the components of the $\mathrm{SN}$, have known reciprocal connectivity with one another. They are also reciprocally connected with motor and sensory areas of the brain. This makes these regions ideally placed to receive the inputs required to initiate switching between the DMN and the CEN. These regions have also shown to be co-activated in different cognitively demanding tasks (Dosenbach et al., 2006). Previous work has also shown that the integrity of white matter from the SN disrupts DMN activation/deactivation (Bonnelle et al., 2012). The importance in understanding these connections comes from previous work suggesting that weak salience detection and mapping of goal-relevant external stimuli and internal mental events from, and into, the SN play a major role in psychopathology (Menon, 2011), and impaired insula, DMN and CEN activity has been linked to psychosis in schizophrenia (Manoliu et al., 2013). Manoliu et al. demonstrated increased functional connectivity between the DMN and CEN in schizophrenia that is related to hallucination severity. In addition the time-lagged connectivity between SN and DMN/CEN was reduced and decreased activity of the salience network was found to be associated with hallucinations and increased functional connectivity between the DMN and CEN.

Sridharan and colleagues highlighted the role of a particular class of neurons present in the salience network that facilitates the network switching process. These neurons are the von Economo neurons, (VENs) which are only found within the salience network (Watson et al., 2006). It has been proposed that "the function of the VENs may be to provide a rapid relay to other parts of the brain of a simple signal derived from information processed within FI and ACC" (Allman et al., 2005). FI is the fronto-insular cortex, comprising the ventro-lateral prefrontal cortex and anterior insula. Sridharan et al. (2008) propose that these neurons constitute the neuronal basis of controlling signals generated by the salience network. Anatomical and neuronal evidence converges to suggest that the brain regions comprising the salience network are ideally positioned for facilitating this network switching. The relationship, demonstrated by Sridharan and colleagues, between the networks is weaker in children, together with weaker structural connectivity. This demonstrates that functional and structural maturation of these pathways is a critical component of the process by which brain networks mature (Uddin et al., 2011).

This work has demonstrated a novel application of DCM using data from an ICA analysis. The analysis presented here could have applications for many different psychiatric and neurological patient groups where network switching may be impaired. The technique of using ICA time courses may be used in many different contexts to investigate the relationships between different networks of the brain. Analyses such as these would complement the functional network connectivity work previously mentioned, as well as other work done comparing connectivity during rest and task conditions (Arbabshirani et al., 2012) in healthy participants and in patients with schizophrenia (Arbabshirani and Calhoun, 2011; White et al., 2010). Using DCM to examine the relationship between the time series will provide further information regarding how these networks interact and switch when a different task is being completed and a different network needs to be recruited.

Differences in the ICA networks between the two datasets are potentially due to the age of the participants. The NITRC dataset comprised healthy young adults, whereas the Bangor dataset comprised some older participants. It has been demonstrated that ageing results in reduced functional connectivity within networks but increased connectivity between networks (Geerligs et al., 2012). It would be of interest to apply this analysis to data from an older population in order to see the effect on the connectivity between the networks and how the network switching is affected. It is worth noting, however, that despite these differences in the cohorts of both groups and the ICA differences, that the results of the BMS are consistent for both datasets. The intrinsic connectivity parameters displayed some significant differences between both datasets however there were no significant differences in the nonlinear modulations.

As mentioned previously these networks are observed when resting state data are decomposed, therefore we believe that these data are useful for studying phenomena such as this. However if a specific network is of interest it would also be useful to have a task that engages that network. An interesting extension of this work would be to perform this analysis on data where a task that engages the salience network is being performed, typically a visual or auditory oddball task. It would then be possible to incorporate bilinear modulations in order to assess the effect of task on the connectivity between these three networks.

In conclusion, we have shown using a novel technique of DCM analysis applied to ICA defined networks it is possible to investigate connectivity and modulation within these networks. In particular, we have independently validated the previous finding that the salience network is involved in the neural process behind switching between DMN and the CEN. We therefore suggest that the techniques discussed in this paper may have widespread use in examining the relationship between different networks of the brain, in a user independent manner, in both healthy individuals and patients with neurological and psychiatric disease. 


\section{Acknowledgments}

The authors would like to acknowledge support from the NeuroSKILL project, a joint Welsh/Irish partnership, part funded by the European Regional Development Fund through the Ireland Wales Programme 2007-13.

\section{References}

Allman, J.M., Watson, K.K., Tetreault, N.A., Hakeem, A.Y., 2005. Intuition and autism: a possible role for Von Economo neurons. Trends Cogn. Sci. 9, 367-373.

Arbabshirani, M.R., Calhoun, V.D., 2011. Functional network connectivity during rest and task: comparison of healthy controls and schizophrenic patients. Conf. Proc. IEEE Eng. Med. Biol. Soc. 2011, 4418-4421.

Arbabshirani, M.R., Havlicek, M., Kiehl, K.A., Pearlson, G.D., Calhoun, V.D., 2012. Functional network connectivity during rest and task conditions: a comparative study. Hum. Brain Mapp. http://dx.doi.org/10.1002/hbm.22118.

Ashburner, J., 2007. A fast diffeomorphic image registration algorithm. NeuroImage 38 95-113.

Beckmann, C.F., DeLuca, M., Devlin, J.T., Smith, S.M., 2005. Investigations into resting-state connectivity using independent component analysis. Philos. Trans. R. Soc. Lond. B Biol. Sci. 360, 1001-1013.

Bell, A.J., Sejnowski, T.J., 1995. An information maximization approach to blind separation and blind deconvolution. Neural Comput. 7, 1129-1159.

Bonnelle, V., Ham, T.E., Leech, R., Kinnunen, K.M., Mehta, M.A., Greenwood, R.J., Sharp, D.J., 2012. Salience network integrity predicts default mode network function after traumatic brain injury. Proc. Natl. Acad. Sci. U. S. A. 109, 4690-4695.

Buckner, R.L., Andrews-Hanna, J.R., Schacter, D.L., 2008. The brain's default network: anatomy, function, and relevance to disease. Ann. N. Y. Acad. Sci. 1124, 1-38.

Chou, Y.H., Panych, L.P., Dickey, C.C., Petrella, J.R., Chen, N.K., 2012. Investigation of longterm reproducibility of intrinsic connectivity network mapping: a resting-state fMRI study. Am. J. Neuroradiol. 33, 833-838.

Comon, P., 1994. Independent component analysis, a new concept. Signal Process. 36, 287-314.

Craddock, R.C., James, G.A., Holtzheimer, P.E., Hu, X.P.P., Mayberg, H.S., 2012. A whole brain fMRI atlas generated via spatially constrained spectral clustering. Hum. Brain Mapp. 33, 1914-1928.

Craig, A.D., 2002. How do you feel? Interoception: the sense of the physiological condition of the body. Nat. Rev. Neurosci. 3, 655-666.

Critchley, H.D., Wiens, S., Rotshtein, P., Ohman, A., Dolan, R.J., 2004. Neural systems supporting interoceptive awareness. Nat. Neurosci. 7, 189-195.

Damoiseaux, J.S., Rombouts, S.A.R.B., Barkhof, F., Scheltens, P., Stam, C.J., Smith, S.M Beckmann, C.F., 2006. Consistent resting-state networks across healthy subjects. Proc. Natl. Acad. Sci. U. S. A. 103, 13848-13853.

Daunizeau, J., Stephan, K.E., Friston, K.J., 2012. Stochastic dynamic causal modeling of fMRI data: should we care about neural noise. NeuroImage 62, 464-481.

Dosenbach, N.U.F., Visscher, K.M., Palmer, E.D., Miezin, F.M., Wenger, K.K., Kang, H.S.C., Burgund, E.D., Grimes, A.L., Schlaggar, B.L., Petersen, S.E., 2006. A core system for the implementation of task sets. Neuron 50, 799-812.

Fox, M.D., Corbetta, M., Snyder, A.Z., Vincent, J.L., Raichle, M.E., 2006. Spontaneous neuronal activity distinguishes human dorsal and ventral attention systems (vol. 103, pg 10046, 2006). Proc. Natl. Acad. Sci. U. S. A. 103, 13560.

Friston, K.J., Harrison, L., Penny, W., 2003. Dynamic causal modelling. Neurolmage 19, 1273-1302.

Geerligs, L., Maurits, N.M., Renken, R.J., Lorist, M.M., 2012. Reduced specificity of functional connectivity in the aging brain during task performance. Hum Brain Mapp.

Hyvarinen, A., Oja, E., 2000. Independent component analysis: algorithms and applications. Neural Netw. 13, 411-430.

Jafri, M.J., Pearlson, G.D., Stevens, M., Calhoun, V.D., 2008. A method for functional network connectivity among spatially independent resting-state components in schizophrenia. Neurolmage 39, 1666-1681.
Lee, M.H., Smyser, C.D., Shimony, J.S., 2012. Resting-state fMRI: a review of methods and clinical applications. AJNR Am. J. Neuroradiol. http://dx.doi.org/10.3174/ajnr.A3263.

Li, B., Daunizeau, J., Stephan, K.E., Penny, W., Hu, D., Friston, K., 2011. Generalised filtering and stochastic DCM for fMRI. NeuroImage 58, 442-457.

Lin, Q.H., Liu, J.Y., Zheng, Y.R., Liang, H.L., Calhoun, V.D., 2010. Semiblind spatial ICA of fMRI using spatial constraints. Hum. Brain Mapp. 31, 1076-1088.

Manoliu, A., Riedl, V., Zherdin, A., Muhlau, M., Schwerthoffer, D., Scherr, M., Peters, H. Zimmer, C., Forstl, H., Bauml, J., Wohlschlager, A.M., Sorg, C., 2013. Aberrant dependence of default mode/central executive network interactions on anterior insular salience network activity in schizophrenia. Schizophr. Bull. 40, 428-437.

Menon, V., 2011. Large-scale brain networks and psychopathology: a unifying triple network model. Trends Cogn. Sci. 15, 483-506.

Menon, V., Uddin, L.Q., 2010. Saliency, switching, attention and control: a network model of insula function. Brain Struct. Funct. 214, 655-667.

Mesulam, M.M., 1998. From sensation to cognition. Brain 121, 1013-1052.

Mesulam, M.M., Mufson, E.J., 1982. Insula of the Old-World Monkey.3. Efferent cortical output and comments on function. J. Comp. Neurol. 212, 38-52.

Mufson, E.J., Mesulam, M.M., 1982. Insula of the Old-World Monkey.2. Afferent cortical input and comments on the claustrum. J. Comp. Neurol. 212, 23-37.

Penny, W.D., Stephan, K.E., Mechelli, A., Friston, K.J., 2004. Comparing dynamic causal models. Neurolmage 22, 1157-1172.

Penny, W.D., Stephan, K.E., Daunizeau, J., Rosa, M.J., Friston, K.J., Schofield, T.M., Leff, A.P., 2010. Comparing families of dynamic causal models. PLoS Comput. Biol. 6, e1000709.

Raichle, M.E., MacLeod, A.M., Snyder, A.Z., Powers, W.J., Gusnard, D.A., Shulman, G.L., 2001. A default mode of brain function. Proc. Natl. Acad. Sci. U. S. A. 98, 676-682.

Rigoux, L., Stephan, K.E., Friston, K.J., Daunizeau, J., 2014. Bayesian model selection for group studies - revisited. NeuroImage 84, 971-985.

Seeley, W.W., Menon, V., Schatzberg, A.F., Keller, J., Glover, G.H., Kenna, H., Reiss, A.L., Greicius, M.D., 2007. Dissociable intrinsic connectivity networks for salience processing and executive control. J. Neurosci. 27, 2349-2356.

Shehzad, Z., Kelly, A.M.C., Reiss, P.T., Gee, D.G., Gotimer, K., Uddin, L.Q., Lee, S.H., Margulies D.S., Roy, A.K., Biswal, B.B., Petkova, E., Castellanos, F.X., Milham, M.P., 2009. The resting brain: unconstrained yet reliable. Cereb. Cortex 19, 2209-2229.

Shirer, W.R., Ryali, S., Rykhlevskaia, E., Menon, V., Greicius, M.D., 2012. Decoding subjectdriven cognitive states with whole-brain connectivity patterns. Cereb. Cortex 22 158-165.

Smith, S.M., Miller, K.L., Salimi-Khorshidi, G., Webster, M., Beckmann, C.F., Nichols, T.E., Ramsey, J.D., Woolrich, M.W., 2011. Network modelling methods for FMRI. NeuroImage 54, 875-891.

Sridharan, D., Levitin, D.J., Menon, V., 2008. A critical role for the right fronto-insular cortex in switching between central-executive and default-mode networks. Proc. Natl. Acad. Sci. U. S. A. 105, 12569-12574.

Stephan, K.E., Kasper, L., Harrison, L.M., Daunizeau, J., den Ouden, H.E., Breakspear, M., Friston, K.J., 2008. Nonlinear dynamic causal models for fMRI. Neuroimage. 42, 649-662.

Stephan, K.E., Penny, W.D., Daunizeau, J., Moran, R.J., Friston, K.J., 2009. Bayesian mode selection for group studies. Neurolmage 46, 1004-1017.

Stevens, M.C., Pearlson, G.D., Calhoun, V.D., 2009. Changes in the interaction of resting-state neural networks from adolescence to adulthood. Hum. Brain Mapp. 30, 2356-2366.

Uddin, L.Q., Supekar, K.S., Ryali, S., Menon, V., 2011. Dynamic reconfiguration of structural and functional connectivity across core neurocognitive brain networks with development. J. Neurosci. 31, 18578-18589.

Watson, K.K., Jones, T.K., Allman, J.M., 2006. Dendritic architecture of the von Economo neurons. Neuroscience 141, 1107-1112.

White, T.P., Joseph, V., Francis, S.T., Liddle, P.F., 2010. Aberrant salience network (bilateral insula and anterior cingulate cortex) connectivity during information processing in schizophrenia. Schizophr. Res. 123, 105-115.

Yu, Q., Sui, J., Rachakonda, S., He, H., Gruner, W., Pearlson, G., Kiehl, K.A., Calhoun, V.D., 2011. Altered topological properties of functional network connectivity in schizophrenia during resting state: a small-world brain network study. PLoS ONE 6, e25423.

Yu, Q., Plis, S.M., Erhardt, E.B., Allen, E.A., Sui, J., Kiehl, K.A., Pearlson, G., Calhoun, V.D. 2012. Modular organization of functional network connectivity in healthy controls and patients with schizophrenia during the resting state. Front. Syst. Neurosci. 5, 103 